\title{
Manifestations of dark energy in the dynamics of the Solar system
}

\author{
Michal Křižek ${ }^{1}$ and Jan Brandts ${ }^{2}$ \\ ${ }^{1}$ Academy of Sciences, Žitná 25, CZ-115 67 Prague 1, Czech Republic \\ email: Krizek@math.cas.cz \\ ${ }^{2}$ Korteweg-de Vries Institute, Faculty of Science, University of Amsterdam, Science Park 904, \\ 1098 XH Amsterdam, The Netherlands \\ email: J.H.Brandts@uva.nl
}

\begin{abstract}
The expansion speed of the Universe is increasing (Glanz 1998). This acceleration is attributed to dark energy which acts almost uniformly everywhere (including the Solar system) and thus essentially influences the Hubble constant. Its current value on a distance of $1 \mathrm{AU}$ is $H_{0}=10 \mathrm{~m} /(\mathrm{yr} \mathrm{AU})$. This is quite a large number and thus, the impact of dark energy should be detectable in the Solar system. We will illustrate it by several examples. Dark energy may partially be caused by gravitational aberration of the Sun, planets and other bodies.
\end{abstract}

Keywords. dark matter, solar system: general, Earth, Moon

1. The Hubble constant. Recent observations suggest that the Hubble parameter $H(t)$ is the sum of a decreasing term $H_{1}(t)=C / t$, which models the Big Bang and subsequent gravitational interaction, and an increasing, but initially relatively small and later more influential term $H_{2}(t)$ of yet unknown origin and nature. The energy needed for the acceleration of the expansion represented by $H_{2}(t)$ is so-called dark energy, which, like dark matter, remains to be detected. The present value $H_{0}$ of the Hubble parameter is about $20 \mathrm{~km} \mathrm{~s}^{-1} \mathrm{Mly}^{-1}$. Since light travels $1 \mathrm{AU}$ in about 500 seconds, we get

$$
H_{0}=\frac{2}{c} \mathrm{~cm} \mathrm{~s}^{-1} \mathrm{yr}^{-1}=0.02 \times 500 /(\mathrm{AU}) \mathrm{m} \mathrm{yr}^{-1}=10 \mathrm{~m} \mathrm{yr}^{-1} \mathrm{AU}^{-1} .
$$

This is quite a large number and therefore, the impact of dark energy should be observable not only on large scales, but also in the Solar system as we shall see.

2. The recession speed of the Moon is larger than accounted for. Laser retroreflectors installed on the Moon reveal that the mean distance $D=384400 \mathrm{~km}$ between Earth and the Moon increases about $3.84 \mathrm{~cm}$ per year. Note that only around $55 \%$ of this value can be explained (Křižek 2009) by tidal forces caused by the Moon and the Sun. The remaining part is often attributed to less explicitly known effects, such as deglaciation, internal processes, and mass transfers in the atmosphere and hydrosphere. An alternative explanation is that the missing part is essentially due to dark energy, which, contrary to the term $H_{1}(t)$, we conjectured to act also locally within the system Earth-Moon. Since the sum $H(t)=H_{1}(t)+H_{2}(t)$ is known to be increasing for already seven billion years, the missing $1.73 \mathrm{~cm}$ per year should ideally be a non-trivial part $H_{2}(T)$ of the total present value $H_{0}=H(T)$ of the Hubble parameter for $T=$ 13.7 Gyr. On the distance $D$ this amounts due to (1) to a yearly expansion of $H_{0}=$ $2.56 \mathrm{~cm} \mathrm{yr}^{-1} D^{-1}$, i.e., $H_{2}(T) \approx 0.67 H_{0}$. Thus, the unexplained part of the recession speed of the Moon is in magnitude indeed comparable to a non-trivial part of the total expansion speed of the Universe. 
3. The ecosphere has been moving, but Earth remained within it. If the current radius of Earth's orbit around the Sun would be more than $5 \%$ larger or $2 \%$ smaller, photosynthesis and hence life as we know it would not be possible. The relatively small region in which life is feasible is called the ecosphere. Obviously, the position of the ecosphere depends on the luminosity of the Sun. Since the Sun is a star of the main sequence, its luminosity has increased approximately linearly during the last 4.5 billion years of its existence, starting at about $70 \%$ of its current value. Therefore, when life originated on Earth 3.5 billion years ago, the luminosity of the Sun was around $77 \%$ of its current value. Since luminosity decreases quadratically with distance, the distance of the ecosphere to the Sun was about $130 \times 10^{6} \mathrm{~km} \approx \sqrt{0.77}$ AU. Thus, Earth must have been much closer to the Sun than it is now, or it would have been frozen, in spite of a different composition of its atmosphere and other temperature increasing effects like a higher level of radioactivity, the impact of comets, and more vulcanism. It is, in fact, actually known that there were liquid oceans on Earth already 4.35 Gyr ago, when the luminosity of the Sun was even smaller. By investigation of fossils of thermophilic bacteria it was found that the temperature of oceans around $80^{\circ} \mathrm{C}$ when life emerged (Lineweaver \& Schwartzman 2003). Taking the above into account, we conclude that Earth must have been around $20 \times$ $10^{6} \mathrm{~km} \approx(1-\sqrt{0.77})$ AU closer to the Sun then it is now. Using this value, we find that the average receding speed of Earth from the Sun to get an almost constant luminosity, and hence influx of solar energy over the past $3.5 \mathrm{Gyr}$, must have been about $5 \mathrm{~m} \mathrm{yr}^{-1}$, i.e., $H_{2}(T) \approx 0.5 H_{0}$ (cf. (1)). Note that Kepler's third law implies that Earth's orbital period increases less than $1 \mathrm{~ms} \mathrm{yr}^{-1}$. This, nor the yearly increase of 5 meters of Earth's distance to the Sun, can be verified by direct measurements. This is particular difficult because due to the influence of the larger planets, the barycentre of the Solar system describes an irregular orbit near the Sun, in which it travels more than $10^{5} \mathrm{~km}$ per year (Bertotti et al. 2003, p. 542). Note that effects like tidal forces from the Sun, the solar wind, and the Sun's decreasing mass are able to explain a recession speed of no more than $0.01 \mathrm{~m} \mathrm{yr}^{-1}$.

The Hubble constant did not change too much during the last $3.5 \mathrm{Gyr}$. It seems that the magnitude of its repulsive part $H_{2}(t)$ is just right for a receding speed giving an almost constant influx of solar energy. This represents further support for the so-called Anthropic Principle, which states that basic physical constants are favorable to the emergence of life only if they are in very narrow intervals.

4. Mars must have been closer to the Sun to have had liquid water. At present, the temperature at the equator of Mars can become close to $0^{\circ} \mathrm{C}$ when the Sun is at its highest point and Mars is at its perihelion, but in average over the day over the whole planet it is with $-63^{\circ} \mathrm{C}$ deeply below the freezing point. Nevertheless, there is convincing evidence for the presence of liquid water in the form of river valleys on Mars around three to four billion years ago. Thus, the average temperature and atmospheric pressure on Mars must have been significantly higher at that time. However, the luminosity of the Sun was then about $73-80 \%$ of its current value, i.e., one third of the solar constant. Moreover, due to snow and ice also in regions away from the polar caps, and due to clouds, the albedo of Mars was higher than at present, implying that more solar energy was reflected back into space. Both facts support the claim that Mars also must have been much nearer to the Sun to have liquid water on its surface $3-4$ Gyr ago. As a consequence we must conclude that Mars have been moving away from the Sun with an average speed of at least several meters per year (cf. (1)).

5. Fast inner satellites have not yet crashed on their mother planet. Each planet has a stationary orbit with radius $R_{\text {stat }}$, where the orbital and rotational periods are equal. Satellites that are below the stationary orbit are called fast. For instance, 
around Uranus there are at least eleven fast satellites and around Neptune five, all between 48 and 76 thousand kilometers away from the centre of their mother planet. All these satellites have nearly circular orbits with small inclinations and orbit their mother planet in the same direction, which indicates that most of them are genuine satellites and have not been captured. Although some may be parts of larger disintegrating satellites, there is no reason to assume that they have not been orbiting their mother planet, below their stationary orbit, for already 4.5 Gyr. However, during this period, tidal forces have acted on them. The resulting tidal bulges continually reduce their potential energy and orbital periods. Thus, all these fast satellites approach their planets along spiral trajectories. Tidal forces are proportional to $M / r^{3}$, where $M$ is the mass of a planet and $r$ is the radius of a given satellite orbit. For all the above-mentioned satellites the value $M / r^{3}$ is of the same order of magnitude as for Phobos, whose orbital period is decreasing. It was estimated that tidal forces are responsible for a decrease of $1.8 \mathrm{~cm} \mathrm{yr}^{-1}$. If we would assume a similar speed 1-2 $\mathrm{cm} \mathrm{yr}^{-1}$ for the other quick satellites, we find that during the last 4.5 Gyr, they must have gotten 45000-90000 km closer to their mother planet. However, all of them (except for Phobos) are in orbits of large radii 0.58-0.92 $R_{\text {stat }}$. This raises the intriguing question why these fast satellites have not fallen down onto their mother planet due to tidal forces. Again, it may be due to dark energy which causes a repulsive force.

6. Kuiper belt. By (Bertotti et al. 2003, p. 534) the Kuiper belt of comets must have been formed much closer to the Sun than its current position indicates. We can easily verify that one half of the value given in (1) is sufficient to have moved this belt over a distance of approximately $10 \mathrm{AU}$ away from the Sun during the last 4.5 Gyr.

7. Delayed Neptune. One half of the value given in (1) would also have shifted Neptune about 5 AU further away from the Sun which explains more easily its formation than at the current region with very low velocities, 30 AU away from the Sun. Moreover, the observed delay of Neptune's position can be partly explained by dark energy.

8. Slow rotation of Mercury. There is also evidence that Mercury may have been closer to the Sun than its current distance of $46-70$ million kilometers. Its sidereal rotation of 59 days is, even for its low mass, very slow. This may be the result of tidal forces that, ten million kilometers closer to the Sun, are twice as large as at its present location, because they decrease cubically with distance. In order to undergo these forces, Mercury must of course have been millions kilometers closer to the Sun than it is now.

9. Conclusions. All of the above strange phenomena may be explained by one source dark energy. This also explains why the system Earth-Moon has at present such a large orbital momentum. From Sect. 2 we know that the distance Earth-Moon increased not only due to tidal forces. In (Kř́ržzek 2009) we illustrate that the dark energy needed for the accelerated expansion of the Universe may partially come from a finite speed of gravitational interaction that causes a positive value of gravitational aberration much smaller than the aberration of light.

\section{Acknowledgements}

The authors thank M. Brož, J. Grygar, and V. Pravda for the useful discussions. Supported by grant no. IAA 100190803 of the Czech Acad. Sci.

\section{References}

Bertotti, B., Farinella, P., \& Vokrouhlický, D., Physics of the Solar System, Kluwer, 2003.

Glanz, J., Science 279 (1998), no. 5355, 1298-1299.

Krrížek, M., Comm. Comput. Phys. 5 (2009), 1030-1044.

Lineweaver, C. H. \& Schwartzman, D., Cosmic thermobiology, In Origins (ed. J. Seckbach), Kluwer, 2003, 233-248. 